\title{
Tratamiento de Agua para Consumo Humano con Alto Contenido de Arsénico: Estudio de un Caso en Zimapán Hidalgo-México
}

\author{
Erasmo Flores $^{1}$, Aurora Armienta ${ }^{2}$ Silvia Micete $^{1}$ y María R. Valladares ${ }^{1}$ \\ (1) Universidad Autónoma Metropolitana-Azcapotzalco, Departamento de Ciencias Básicas, Área de \\ Química, Av. San Pablo No. 180, 02200 México-D.F.-México (e-mail: efv@correo.azc.uam.mx) \\ (2) Universidad Nacional Autónoma de México, Instituto de Geofísica, Circuito exterior S/N, Zona \\ de Institutos, Cd. Universitaria, 04510 México, D.F.-México
}

\begin{abstract}
Resumen
El presente estudio tiene por objeto demostrar que las propiedades fisicoquímicas de la roca Caliza Soyatal, localizada en la región de Zimapán Hidalgo (México), facilitan los niveles de adsorción de arsénico cuando la roca se pone en contacto con el agua contaminada, reduciendo considerablemente las concentraciones de dicho metal. Aunque la roca Soyatal contiene arsénico, las pruebas de desorción efectuadas indican niveles mínimos de dicho fenómeno. Las pruebas se realizaron usando torres empacadas con la roca Soyatal para eliminar el arsénico presente en el agua. Durante las pruebas de laboratorio se consideraron ciertas variables que determinan la eficiencia de remoción de arsénico: tamaño de partícula, tiempo de contacto, cantidad de roca y volumen de la muestra. A partir de los resultados, se puede concluir que esta investigación puede tener utilidad para implementar un tratamiento casero para tratar el agua de consumo humano proveniente del pozo Zimapán.
\end{abstract}

Palabras clave: arsénico, tratamiento de agua, roca caliza, desorción, adsorción

\section{Water Treatment for Human Consumption with High Arsenic Content: A Study Case in Zimapán Hidalgo- Mexico}

\begin{abstract}
The present study intends to demonstrate that physical and chemical properties of the Limestone rock Soyatal, located in the region of Zimapán Hidalgo (Mexico) facilite the levels of arsenic adsorption when the rock is put into contact with contaminated water, reducing considerably the concentrations of this metal. Although Soyatal rock contains arsenic, desorption tests were done with the same rock, finding a minimum occurrence of this phenomenon. Tests were done using towers packed with Soyatal rocks to eliminate the arsenic present in the water. During the laboratory tests certain variables were considered to determine the efficiency of arsenic removal: particle size, contact time, amount of rock and volume of the sample. From the results it can be concluded that this study can be useful for implementing a domestic treatment to purify drinkable water extracted from the Zimapán well.
\end{abstract}

Keywords: arsenic, water treatment, limestone rock, desorption, adsorption 


\section{INTRODUCCIÓN}

Los cuerpos de agua subterráneos han sido estudiados desde hace tiempo debido a problemas presentados, particularmente, por la contaminación de arsénico en aguas subterráneas en diversas zonas en el mundo. Se han encontrado concentraciones de arsénico muy elevadas en aguas subterráneas destinadas al consumo humano en Nueva Escocia, Canadá y algunos otros países, las cuales se consideran, son producto del intemperismo en pilas de desechos mineros que contienen arsenopirita (Bech et al.,1997; Barnes y Erickson, 2005). En algunos casos, la presencia de arsénico es consecuencia de contaminación natural y en otros por contaminación antropogénica causada principalmente por las actividades agrícolas y ganaderas. En el oeste de los Estados Unidos es común encontrar altas concentraciones de arsénico en las aguas subterráneas asociadas con áreas de actividad geotérmica y minera (Fetter, 1999). En Alemania la contaminación se la adjudica a la actividad agrícola, esta hipótesis deriva de la presencia de arsénico en las uvas (Bates et al., 1992; Bolan et al., 2007).

En México se han reportado altas concentraciones de arsénico en varios lugares como es el caso del Valle de Guadiana, Sonora (Alarcón, 2001), que presentó concentraciones de 40 a 136 por ciento sobre los niveles permisibles y el de la Comarca Lagunera, que presentó un intervalo de 0,24 a 1,0 $\mathrm{mg} / \mathrm{L}$ (Olguín et al., 1983). También se reporta contaminación de Arsénico en los estados de Coahuila, Durango y Aguascalientes (Trejo y Bonilla, 2002).

Las investigaciones en México se han dirigido principalmente a lugares donde se detectan los mayores problemas de intoxicación y/o epidemias. Uno de los casos más relevantes es el de Zimapán Hidalgo, en donde, al alertarse de una epidemia de cólera en el país, se realizó un estudio que determinó altos índices de arsénico en el agua. En el estudio realizado por Armienta (1993), reportó una concentración de 1,097 mg/L en uno de los pozos, lo cual indicó que se encontraba fuera del límite máximo permitido para el agua potable que era de 0,05 mg/L conforme a la NOM-127SSA1-1994. Actualmente es de 0,025 mg/L, conforme a la NOM-127- SSA1-1994-2000 (1994)

En este municipio, se han realizado estudios biomédicos en la población debido a la intoxicación crónica que se ha presentado por la ingestión del agua potable contaminada con arsénico. Para este estudio se tomaron muestras de pelo de 120 habitantes, también se aplicó un cuestionario donde se incluyeron datos generales sobre la información de la historia familiar, en padecimientos de cáncer, hígado, enfermedades respiratorias y de la piel principalmente, se excluyeron las personas que trabajaban en las minas y fundidoras. Los niveles normales de arsénico en el pelo son de 0,3 a 1,75 $\mathrm{mg} / \mathrm{kg}$ y en Zimapán la concentración promedio fue de 8,55₫3,56 mg/kg (Armienta et al., 1997; Gómez-Arrollo et al., 1997).

Debido a que la fuente principal de intoxicación por arsénico en esta población se debe a la ingestión del agua potable, una de las principales medidas preventivas consiste en la adecuada potabilización del agua para su consumo. La tasa máxima permitida de arsénico para el agua potable es de 0,025 mg/L según la norma oficial mexicana NOM-127-SSA1-1994-2000 (1994) "Salud ambiental, agua para uso y consumo humano-límites permisibles de calidad y tratamiento que debe someterse al agua para su potabilización". Se ha demostrado que el agua que ingiere la población como agua potable en Zimapán Hidalgo no tiene la calidad que exige la normatividad vigente.

Según estudios de interacción de roca-agua reportan que algunas rocas pueden adsorber arsénico en sus capas, el porcentaje de adsorción depende de la cantidad de roca-agua que estén en contacto (Romero, 2000).

El presente estudio tiene por objeto demostrar que las propiedades fisicoquímicas de la roca caliza Soyatal localizada en la región de Zimapán Hidalgo, facilitan la de adsorción de arsénico cuando se ponen en contacto con el agua contaminada, reduciendo considerablemente las concentraciones de dicho metal en el agua. Un tratamiento casero sugerido por Ongley et al. (2001), consiste en colocar roca caliza en una porción de $100 \mathrm{~g}$ por litro de agua contaminada, agitar manualmente y decantar, esta porción de roca podría utilizarse durante cinco ciclos. 


\section{DESARROLLO EXPERIMENTAL}

\section{Muestreo de agua}

Por un lado se observó que existe una baja cantidad de agua superficial, por el otro, la existencia de agua subterránea, razón por la cuál la gente construye norias (pozos de poca profundidad $<30 \mathrm{~m}$ ) que explotan para servicio de autoconsumo.

El $1^{\text {er }}$ muestreo se realizó en octubre del 2001, este estudio se llevó a cabo en el pozo Zimapán V, conforme a la NOM 014-SSA1 (1993). En dicha norma se enuncian los parámetros a determinar y la forma correcta de llevar el muestreo, conservación y manejo de las muestras hasta su ingreso al laboratorio. La determinación de arsénico se llevó a cabo siguiendo la norma NMX-AA-051-SCFI (2001), la cual indica el método espectrofotométrico de absorción atómica con generación de hidruros para determinar arsénico.

Se realizó un segundo muestreo en mayo del 2002 en varios pozos. Posteriormente, se realizaron otros muestreos en abril de 2003, octubre de 2003 y en febrero de 2005.

\section{Muestreo de roca}

Se definió el tipo de roca según sus características. En la búsqueda bibliográfica se encontraron varios tipos, sin embargo, la que parece más viable es la formación Soyatal que tiene un mayor porcentaje de calcita, que en teoría, es un buen medio adsorbente.

El lugar de muestreo se encuentra dentro del municipio de Zimapán Hidalgo. Se llevó a cabo de la siguiente manera: se identificó el lugar, se seleccionó la roca in situ, se probó de campo, se colectó en bolsas de polietileno, se transportó al laboratorio, se secó (en condiciones ambientales), se molió, tamizó, y selecciono por tamaño, se aplicó el método de cuarteo para cada tamaño. Para identificar las propiedades de las rocas recolectadas en campo se les agregaron unas gotas de $\mathrm{HCl}$, se observó un burbujeo por generación de $\mathrm{CO}_{2}$ que se produce conforme a la reacción:

$\mathrm{CaCO}_{3(\mathrm{~s})}+2 \mathrm{HC}_{\mathrm{l}(\mathrm{ac})} \rightarrow \mathrm{CaCl}_{2(\mathrm{~s})}+\mathrm{H}_{2} \mathrm{O}_{(\mathrm{l})}+\mathrm{CO}_{2(\mathrm{~g})}$

Ésta es una de las formas utilizada para asegurar que dicha muestra contiene el componente necesario.

\section{Análisis de arsénico en la roca caliza Soyatal}

El siguiente paso consistió en el análisis de roca para determinar si contiene arsénico. El procedimiento fue el siguiente: Se colocó $1 \mathrm{~g}$ de muestra de roca Soyatal, y se agregaron $10 \mathrm{ml}$ agua regia. El agua regia se prepara mezclando 3 partes de ácido clorhídrico concentrado y una parte de ácido nítrico concentrado. La función de la digestión con ácido clorhídrico, ya sea en sistema frío o con temperatura elevada es descomponer los carbonatos y rocas carbonatadas; por su parte, la función del ácido nítrico es descomponer algunos minerales sulfatados presentes. Ésta función puede ser la más importante aplicación en análisis de rocas y también se usa para la determinación de metales pesados presentes en los sólidos, toda vez que se forman los nitratos metálicos solubles. A continuación se evaporó lentamente hasta sequedad, se disolvió con $\mathrm{HCl}$ y se llevó al volumen original con agua deionizada, se filtró y se analizó por absorción atómica con generación de hidruros. Previamente se prepararon los estándares para la curva de calibración, se registraron las lecturas de absorbancia para obtener la gráfica de calibración correspondiente, en la cual se interpolan las absorbancias que se obtienen de las muestras. En la realización de estos análisis se utilizó un equipo Perkin Elmer 2380 con generación de hidruros y se siguió la NOM-051-SCFI-2001.

\section{Pruebas de desorción de arsénico de roca caliza Soyatal}

Estas pruebas se realizaron en torres empacadas con roca caliza Soyatal con un flujo de agua normal, en continuo (figura 1) y otro con recirculación (figura 2). El mismo procedimiento se aplicó en ambas torres. 


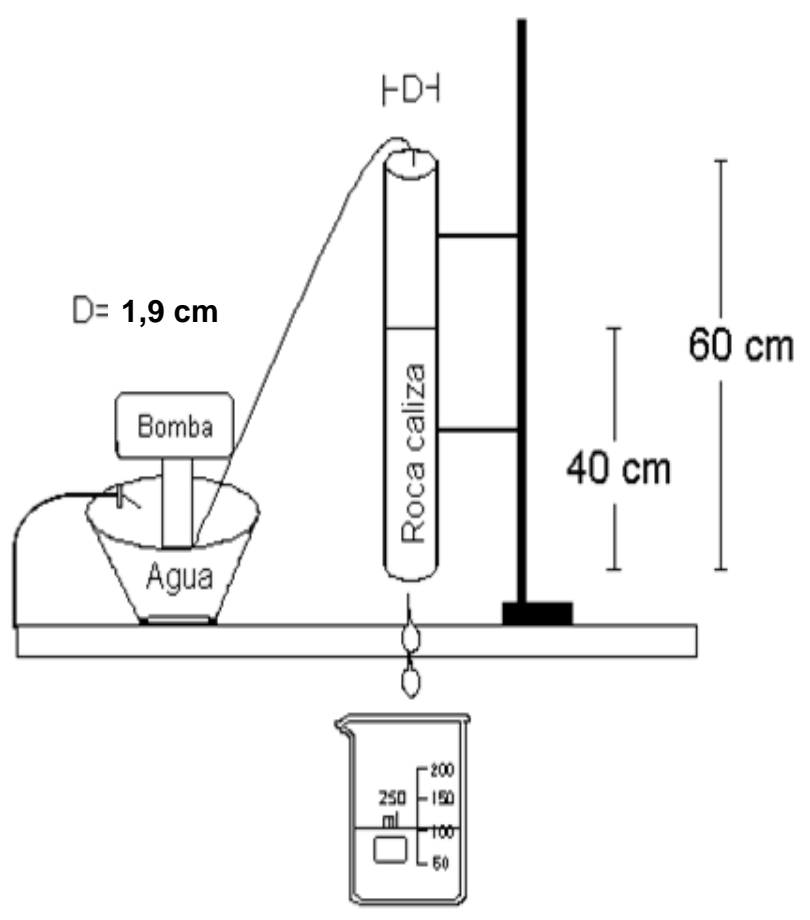

Fig 1: Esquema de la desorción en sistema continuo.

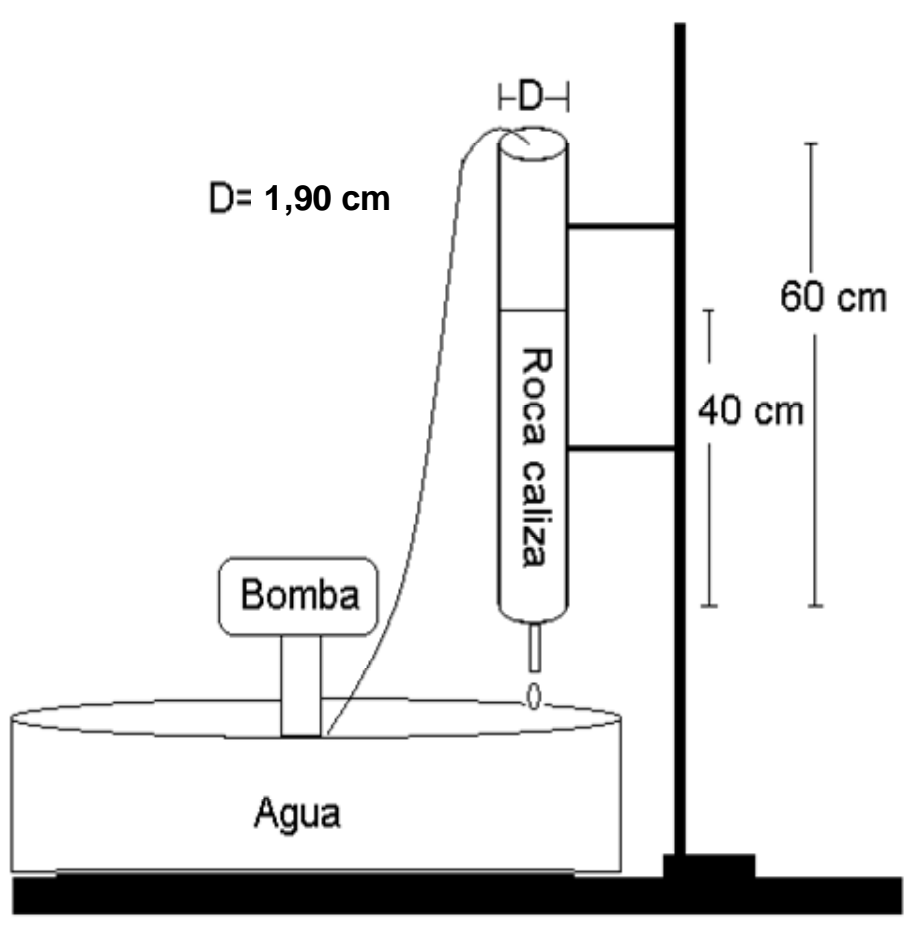

Fig 2: Esquema de la desorción en sistema con recirculación.

Prueba de adsorción de arsénico en Sistema Batch en roca caliza de diámetro 0,5 mm

En vasos de precipitado de $250 \mathrm{ml}$, por separado, se colocaron 1, 5 y 10 gramos de roca caliza Soyatal y $100 \mathrm{ml}$ de agua del pozo Zimapán V, se pusieron en agitación, se filtraron, el filtrado en cada caso se analizó por espectrometría de absosrción atómica (Kumar y Riyazuddin, 2008) con generación de hidruros utilizando un equipo Perkin Elmer 2380, las absorbancias registradas se 
interpolaron en una curva de calibración previamente preparada, utilizando una solución estandar de arsénico marca Merck, el coeficiente de correlación obtenido de la curva de calibración fue de $r$ $=0,998$.

\section{RESULTADOS Y DISCUSIÓN}

El valor obtenido de Arsénico en el muestreo del agua se comparó con el límite máximo permisible establecidos por la NOM-127-SSA1-1994-2000 (1994), que es de 0,025 mg/L. Los resultados se comparan con los de la literatura mencionada como se muestra en la tabla 1.

Tabla 1: Comparación de concentración de arsénico en el pozo Zimapán V.

(Tomado de Armienta et al., 1997)

\begin{tabular}{|c|c|c|c|c|c|c|c|c|c|c|c|c|c|c|}
\hline Año & $\mathrm{Eh}(\mathrm{mV})$ & $\mathrm{T}\left({ }^{\circ} \mathrm{C}\right)$ & $\mathrm{pH}$ & $\mathrm{SO} 4$ & $F$ & $\mathrm{Na}$ & $\mathrm{K}$ & $\mathrm{Cl}$ & As & \begin{tabular}{|l|}
$\mathrm{HCO} 3$ \\
\end{tabular} & $\mathrm{COB}$ & $\mathrm{Ca}$ & $M g$ & $\mathrm{Fe}$ \\
\hline 1997 & 147 & 29 & 7,82 & 62,3 & 0,7 & 10 & 1,7 & 4 & 0,53 & \begin{tabular}{|l|}
270,7 \\
\end{tabular} & 0 & 87 & 13 & 0,5 \\
\hline 2001 & 483 & 28,5 & 7,8 & 106 & 0,7 & 11 & 1,6 & 5 & 0,52 & 263,49 & 0 & 79 & 18 & $\star \star \star \star \lambda$ \\
\hline 2002 & & & & & & & & & 0,36 & & & & & \\
\hline 2003 & & & & & & & & & 0,46 & & & & & \\
\hline 2003 & & & & & & & & & 0,5 & & & & & \\
\hline 2005 & & & & & & & & & 0,5 & & & & & \\
\hline
\end{tabular}

Se observa que los valores de la concentración de Arsénico están fuera de norma y son similares entre ellos, aunque tienen algunas diferencias que se pueden atribuir a la oxidación de los minerales del arsénico al tener contacto con el oxígeno que entra ya sea por efectos climatológicos impactando en las aguas subterráneas o por efectos de operación directa en el pozo (figura 3)

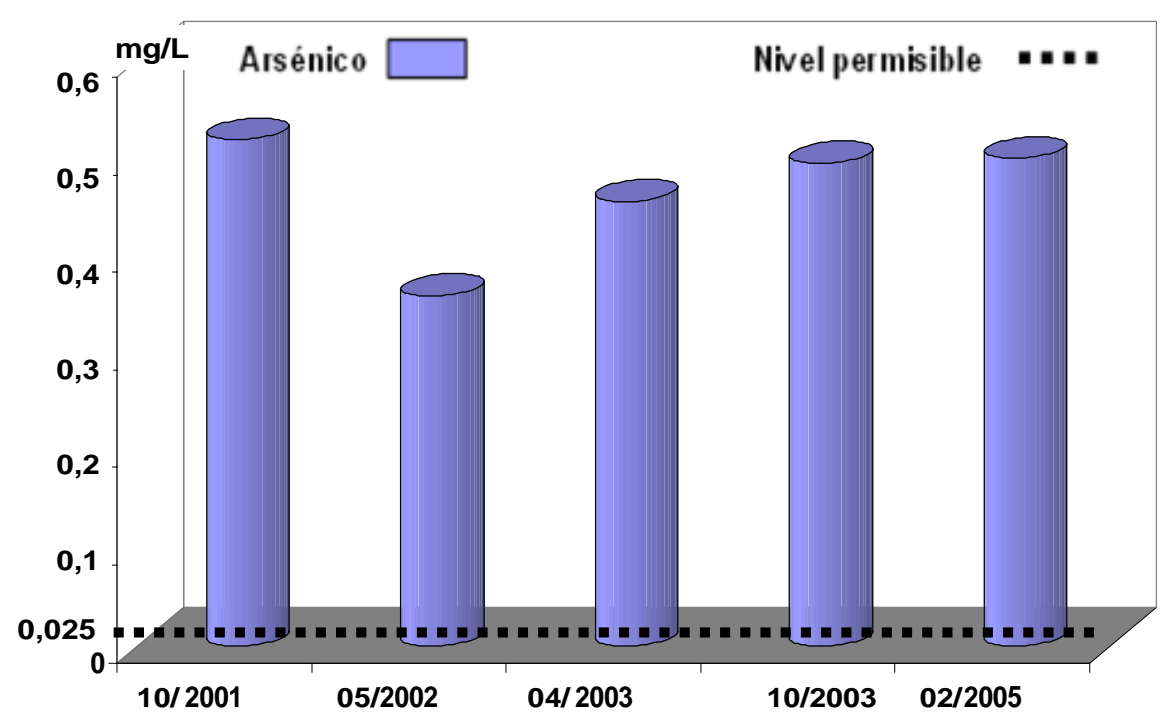

Fig. 3: Comparación de concentración de arsénico en el pozo Zimapán $\vee$ y nivel permisible

En el análisis de arsénico en roca caliza Soyatal, se pudo comprobar que esta roca caliza contiene arsénico, pero no está saturada y aun tienen capacidad de adsorción. Los resultados se muestran en la tabla 2.

Los resultados obtenidos de la concentración de arsénico en la roca caliza indican que este material presenta capacidad para adsorber arsénico, dichos resultados experimentales corroboran investigaciones anteriores acerca de interacciones roca agua en las cuales reportan que en sus capas se adsorbe arsénico y que el porcentaje de adsorción depende de la relación roca- agua que esté en contacto (Romero, 2000;, Ongley et al., 2001; Berner et al., 2008) la capacidad de adsorción de la roca es atribuida a su composición química, dada por los minerales, arcilla, cuarzo y calcita, 
esta última como componente en predominio (Cornelius et al., 1992). En estas propiedades de la roca se basa un tratamiento casero sugerido por Ongley et al. (2001).

Tabla 2: Resultados obtenidos de la concentración de Arsénico en la roca caliza Soyatal.

\begin{tabular}{|c|c|}
\hline \multicolumn{2}{|c|}{ Roca caliza Soyatal } \\
\hline $\begin{array}{l}\text { Tam año } \\
(\mathrm{m} \mathrm{m})\end{array}$ & $\begin{array}{l}\text { Concen tración } \\
\text { de As }(\mathrm{m} \mathrm{g} / \mathrm{kg})\end{array}$ \\
\hline$>2$ & 33,8 \\
\hline $0,7-2,0$ & 33,15 \\
\hline $0,43-0,7$ & 40,8 \\
\hline $0,05-0,43$ & 32,5 \\
\hline$<0,05$ & 27,3 \\
\hline
\end{tabular}

Los procesos de desorción de arsénico en sistemas continuo y con recirculación demostraron que no existen grandes diferencias entre los dos procesos, las variaciones que se observaron, se pueden atribuir a las variaciones de flujo debido a la intervención de factores operativos. Después de los 50 minutos se observa una mayor desorción en el sistema continuo (figura 4).

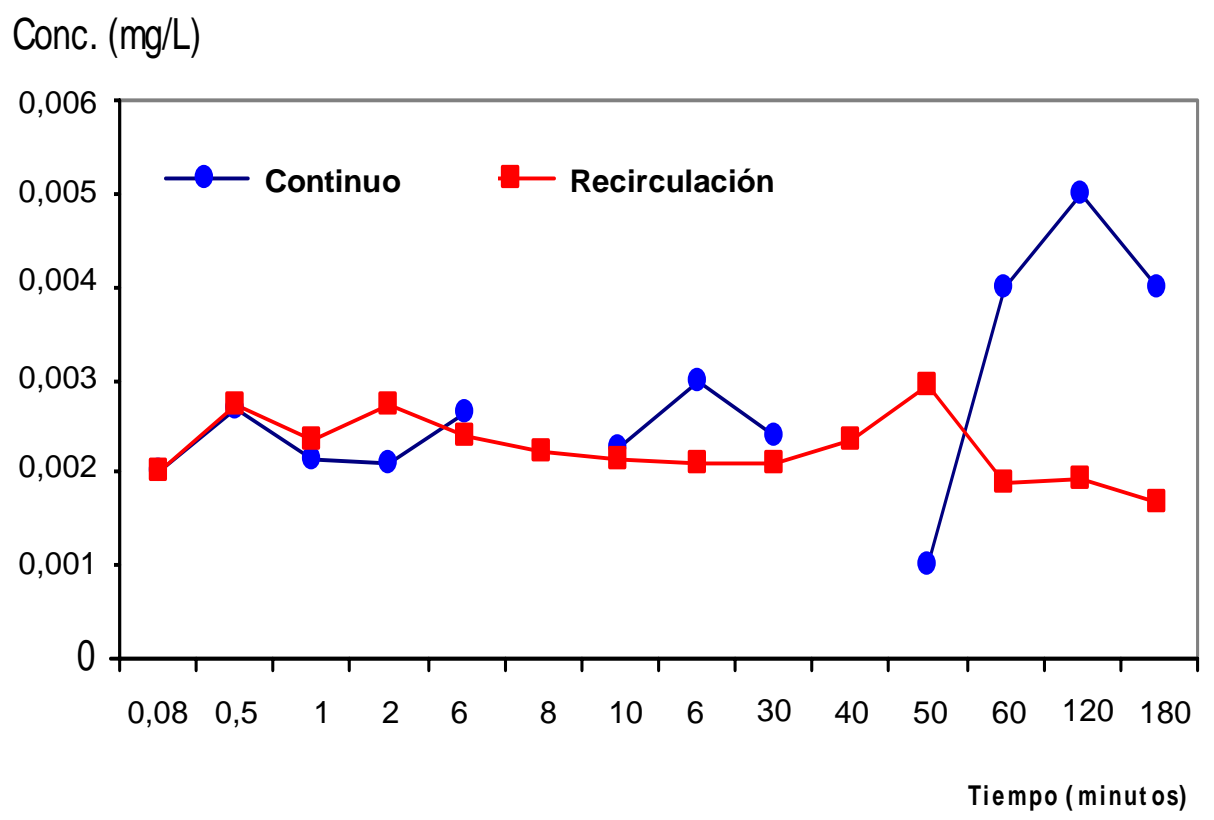

Fig 4: Comparación de los resultados de la desorción en sistema continuo y con recirculación.

Los resultados de esta prueba indican que el arsénico contenido en las rocas no se libera al ponerse en contacto con el agua con lo cual se determina que no existen niveles de desorción considerables. Lo anterior puede explicarse considerando que los enlaces entre el adsorbato y el adsorbente son fuertes, semejantes a los enlaces químicos y el proceso casi siempre es irreversible, a diferencia de una adsorción física, donde los enlaces son débiles semejantes a las fuerzas de Van der Waalls.

En la tabla 3 se muestran los resultados de eficiencia de remoción de arsénico por la roca caliza Soyatal cuando varían las cantidades de ésta y el volumen de muestra es constante.

Se presentan resultados de la roca de $0,5 \mathrm{~mm}$ de diámetro, aunque se hicieron experimentos con rocas de $0,25,0,84$ y $1 \mathrm{~mm}$ de diámetro (Micete, 2005) la roca de $0,5 \mathrm{~mm}$ presenta remociones muy semejantes a la de $0,25 \mathrm{~mm}$ y tiene la ventaja de menos apelmasamiento en su manejo. El tamaño de la roca determina en gran medida la capacidad de remoción del adsorbato, la cual se incrementa en la medida que decrece el diámetro promedio de las partículas adsorbentes. Esto se debe a una 
mayor área de adsorción disponible en el caso de partículas pequeñas (Jerome, 1994; Ayoob et al., 2006).

Tabla 3: Resultado de adsorción de arsénico en roca caliza 0,5 mm de diámetro con $100 \mathrm{ml}$ de agua del pozo Zimapán $\mathrm{V}$.

\begin{tabular}{|c|c|c|c|c|c|c|c|c|c|}
\hline & \multicolumn{3}{|c|}{$1 \mathrm{~g}$} & \multicolumn{3}{c|}{$5 \mathrm{~g}$} & \multicolumn{3}{c|}{$10 \mathrm{~g}$} \\
\hline $\begin{array}{c}\text { tiempo } \\
\mathrm{h}\end{array}$ & $\begin{array}{c}\text { Conc } \\
(\mathrm{mg} / \mathrm{l})\end{array}$ & $\begin{array}{c}\text { Desv } \\
\text { Est }\end{array}$ & Eficiencia & $\begin{array}{c}\text { Conc. } \\
(\mathrm{mg} / \mathrm{l})\end{array}$ & $\begin{array}{c}\text { Desv } \\
\text { Est. }\end{array}$ & Eficiencia & $\begin{array}{c}\text { Conc } \\
(\mathrm{mg} / \mathrm{l})\end{array}$ & $\begin{array}{c}\text { Desv } \\
\text { Est }\end{array}$ & Eficiencia \\
\hline 0 & 0,456 & $\pm 0,0009$ & & 0,456 & $\pm 0,0009$ & & 0,456 & $\pm 0,0009$ & \\
\hline $1 \mathrm{~h}$ & 0,0452 & $\pm 0,0054$ & 90 & 0,0260 & $\pm 0,0042$ & 94,29 & 0,0160 & $\pm 0,0028$ & 96,5 \\
\hline $3 \mathrm{~h}$ & 0,0520 & $\pm 0,0007$ & 88,6 & 0,0246 & $\pm 0,0011$ & 94,60 & 0,0150 & $\pm 0,0006$ & 96,71 \\
\hline $5 \mathrm{~h}$ & 0,0481 & $\pm 0,0098$ & 89,45 & 0,0183 & $\pm 0,0030$ & 96 & 0,0140 & $\pm 0,0004$ & 97 \\
\hline
\end{tabular}

En el experimento con el que se trabajó con $10 \mathrm{~g}$ de roca se alcanzó hasta un 96,50 por ciento de remoción de arsénico. También se pudo comprobar que la remoción se lleva a cabo en la interacción inicial del agua con la roca y posteriormente se estabiliza (figura 5). Este experimento se realizó a diferentes tiempos ( 1,3 y 5 horas ) y cantidades ( $1 \mathrm{~g}, 5 \mathrm{~g}$ y $10 \mathrm{~g}$ ) disueltas en $100 \mathrm{ml}$ de agua, con el objeto de encontrar la cantidad de roca y tiempo necesario para una mayor remoción de arsénico.

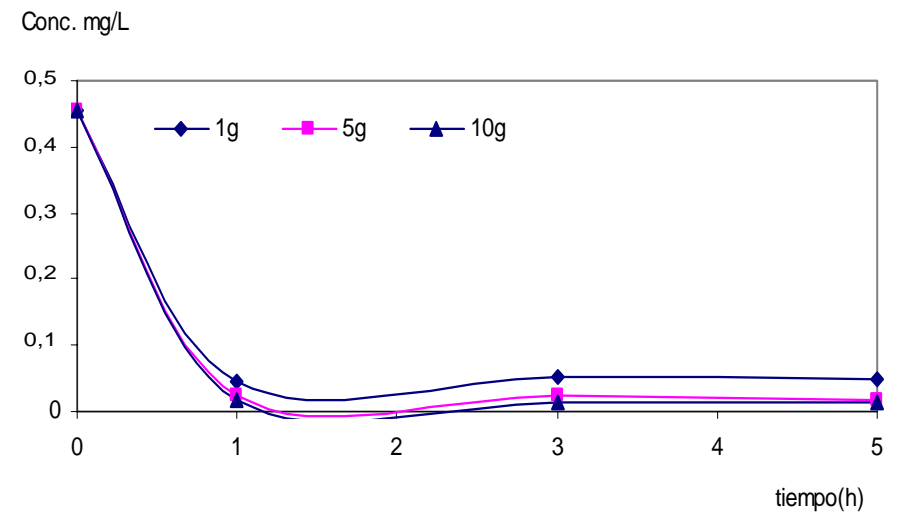

Fig 5: Resultado comparativo de adsorción de arsénico en roca caliza Soyatal de 0,5 mm con100 ml de agua de pozo Zimapán $\mathrm{V}$ a diferentes tiempos y cantidades de roca.

\section{CONCLUSIONES}

La caracterización del agua del pozo Zimapán $\vee$, ubicado en Zimapán Hidalgo., en diferentes fechas corrobora la presencia de concentraciones uniformes del arsénico.

Existen ventajas en la región estudiada, ya que el medio filtrante (roca caliza Soyatal) se puede encontrar en dicha área.

Aunque la roca caliza contiene arsénico, las concentraciones son bajas y con pruebas experimentales queda demostrado que dicha especie no presenta niveles considerables de desorción.

Esta investigación puede tener utilidad para implementar un tratamiento casero para tratar el agua de consumo humano proveniente del pozo Zimapán $\mathrm{V}$.

\section{REFERENCIAS}

Alarcón, M.; Contenido de arsénico en el agua potable de Valle de Guadiana, Ingeniería Hidráulica en México: 16 (4), 63-70 (2001). 
Armienta, M.A.; Estudio de reconocimiento de la contaminación por arsénico en la zona de Zimapán, Hidalgo. Reporte técnico, IGF-UNAM, México (1993).

Armienta, M.A., R. Rodriguez. y O. Cruz; Arsenic content in hair of people exposed to natural arsenic polluted groundwater at Zimapan, Bull. Environ. Contam. Toxicol.: 9, 583-589 (1997).

Ayoob, S., P.B. Bhakat., A.K. Gupta y S. Kundu; Investigations on arsenic (V) removal by modified calcined bauxite, Colloids \& Surfaces A: Phys. Eng. Asp.: 281(1-3), 237-245 (2006).

Barnes, R. J. y M.L. Erickson; Well characteristics influencing arsenic concentrations in ground water, Water Research: 39 (16) 4029-4039 (2005).

Bates, M.N., A.H. Smit y C. Hopenhayn-Rich; Arsenic ingestion and internal cancers. American Journal of Epidemiology: 135 (5), 462-466 (1992).

Bech, J. y otros 7 autores; Arsenic an heavy metal contamination of soil and vegetation around a copper mine in Northern Peru. The science of the total environment: 203, 83-91 (1997).

Berner, Z.G., Huaming Staiben y D.U. Kramar; Adsorption of arsenic species from water using activated sideriteâ€"hematite column filters, Journal of Hazardous Materials: 151(2/3), 628-635 (2008).

Bolan, N.S. y otros 5 autores; Mobility of copper, chromium and arsenic from treated timber into grapevines, Science of the Total Environment: 388 (1-3), 35-42 (2007).

Cornelius, S., Jr. Hurlbut y K. Cornelius; Manual de Mineralogía. Ed. Reverté, Barcelona (1992)

Fetter, C.W.; Contaminant Hidrogeology. Macmillan Publishing Company, N.Y. (1999).

Gomez-Arroyo S., M.A. Armienta., J. Cortes-Eslava y R. Villalobos-Pietrini; Sister-Chromatid Exchanges in Vicia-Faba Induced by Arsenic-Contaminated Drinking Water From Zimapán Hidalgo Mexico. Mutation Research Genetic Toxocology and Enviromental Mutagenesis: 394, 1-7 (1997).

Jerome, O.; Arsenic in the environment, Vol. 27, Johns Wiley and Sons, Inc, USA (1994).

Kumar, A.R. y P. Riyazuddin; Determination of arsenic(III) and total inorganic arsenic in water samples using variable tetrahydroborate(III) and acid concentrations by continuous-flow hydridegeneration atomic absorption spectrometry, International Journal of Environmental Analytical Chemistry: 88(4), 255-266 (2008).

Micete Flores, S., "Diseño de una planta piloto basada en adsorción en rocas calizas para el tratamiento del agua contaminada con arsénico del pozo Zimapán $V$ en el Municipio de Zimapán, Hidalgo" Tesis de grado, Dpto. de Ciencias Básicas, Universidad Autónoma Metropolitana, Unidad, Azcapotzalco, México, D.F. (2005).

Olguín, A., A. Albores., M. Cebrián y P. Jauge; Arsenic levels in blood, urine, hair, and nails from a choronically exponed human population. Proc. West. Pharmocol. Soc,: 26,175-177 (1983).

Ongley, L. y otros 5 autores; Arsenic removal from contaminated water by the Soyatal Formation, Zimapán Mining District, Mexico - a potential low-cost low-tech remediation system, Geochemistry Exploration, Environment Analysis: 1(1), 23-31 (2001).

Romero, F.; "Interacción de aguas contaminadas con arsénico con rocas calizas de Zimapán, Hidalgo", Tesis de grado, Instituto de Geofísica. UNAM. México (2000).

Trejo Vázquez, R. y A. Bonilla Petriciolet; Cuantificación de As en el agua subterránea de la ciudad de Aguascalientes, México y elevación de riesgos entre la población. Ingeniería Hidráulica en México: 4(18), 78-89 (2002). 
NOM-127-SSA1-1994-2000; Norma Oficial Mexicana sobre Salud ambiental, agua para uso y consumo humano-límites permisibles de calidad y tratamiento que debe someterse al agua para su potabilización, México D.F.(1994)

NOM 014-SSA1, Norma Oficial Mexicana sobre parámetros a determinar y la forma correcta de llevar el muestreo, conservación y manejo de las muestras hasta su ingreso al laboratorio, México D.F. (1993).

NMX-AA-051-SCFI-2001, Norma Oficial Mexicana que indica el método espectrofotométrico de absorción atómica con generación de hidruros para determinar arsénico, México D.F. (2001). 
